\title{
An Efficient Testing for the Detection of Trajectories in Discrete-Event Systems Modelled by S-Nets
}

\author{
R. CAMPOS-RODRIGUEZ*, M. ALCARAZ-MEJIA \\ ITESO University, \\ Periferico Sur \# 8585, Tlaquepaque, 45604, Mexico, \\ rcampos@iteso.mx, mildreth\}@iteso.mx
}

\begin{abstract}
This paper addresses the problem of the detection of sequences of event executed in a Discrete-Event System modelled by Petri Nets. The nets are equipped with output symbols that an external observer is allowed to detect. To provide efficient solutions, the focus of this work is a subclass of nets called S-Systems. The construction of the Sequence-Detectability table leads to a necessary and sufficient condition for the characterization of the sequence detection in the case of safe nets. The safeness requirement is relaxed and its implication on the sequence detection is analyzed. Moreover, the utility of the sequence detectability in the analysis of the observability of the net is studied. An example illustrates the concepts and main results of this paper.
\end{abstract}

Keywords: Discrete-Event Systems, System Trajectories, Sequence-Detection, Petri Nets, Observability, S-systems.

\section{Introduction}

The detection of the trajectories in a system is important for several purposes. For example, in Discrete-Event Systems (DES) some control schemes and observer designs use the sequence of events as feedback, while several techniques for the failure detection employ the system trajectories to establish a suitable recovery point [13], [16], [18], [19], [22].

Several approaches reported in the literature dealing with the formal analysis of DES have studied the system trajectories as part of other problems. For instance, the design of controllers, observers, trackers and detectors for DES have all been studied within the automaton field [10], [15], [19], [20], [21].

Petri nets (PN's) have also been used for the analysis of almost the same problems as in the automaton framework. For example, the modeling of manufacturing systems, the supervisory control, state feedback schemes, sequence detection and the observability of PN's and the design of controllers and observers, have been reported in [1], [5], [6], [7], [9], [11], [16].

Previous related work of the authors of this paper is also reported in the literature. In [2], a technique that allows controlling a DES using a supervisor with the feedback provided by an observer of sequences is developed. In [3], preliminary results for addressing the sequence detection of a subclass of PN, called FreeChoice nets, are provided.
This paper extends the previous research of the authors and provides novel results and efficient algorithms addressing the problem of the sequence detection in DES that are modelled by S-Systems, a subclass of PN with well-defined structure, where efficient solutions are derived. Firstly, safe nets are considered. For this case, the construction of the Sequence-Detectability table provides a necessary and sufficient condition for the testing of the sequencedetectability. Secondly, the safeness requirement is relaxed and its implication on the Sequence-Detectability is analyzed. For the non-safe case, the construction of the EventDetectability table provides a necessary and sufficient condition for the verification of the property. Finally, the relationship among the sequence-detectability and the observability of S-Systems is outlined.

The rest of this paper is organized as follows. Section 2 introduces some basic PN's notions used in this work. Section 3 presents the Sequence-Detectability property and its characterization in well-formed S-Systems. Section 4 outlines how the sequencedetectability supports solving the observability of the net. Section 5 provides the conclusions of this work and a final section shows the bibliographical references.

\section{Petri Nets Preliminaries}

This work uses Output Petri nets (OPN's) for representing a DES. An OPN is similar to a PN [13] but it is equipped with an output function that produces vectors of output 
signals. For more details of OPN's see [3]. Formally, an OPN is a tuple $\left(B, M_{0}, \varphi\right)$ with the following properties:

- $B$ stands for a standard Petri Net Structure (PNS) $\{P, T, I, O\}$, where $P$ is a set of places, $T$ is a set of transitions; $I \subseteq P \times T$ and $O \subseteq P \times T$ are the flow relations;

- $\quad M_{0} \in\left(\mathbb{N}^{+}\right)^{m}$ is a special vector known as the initial marking, where $m=|P|$;

- The linear function $\varphi:\left(\mathbb{N}^{+}\right)^{m} \rightarrow\left(\mathbb{N}^{+}\right)^{q}$ that relates a marking vector to output signals, where $q$ is the number of sensors.

The output function is represented a matrix $\varphi_{[q \times m]}$, where the $i-t h$ row vector $\varphi(i,:)$ represents the places associated to the $i-t h$ output signal. That is, if $p_{j} \in P$ is associated with the $i$-th sensor, then $\varphi(i, j)=1$, otherwise $\varphi(i, j)=0$. An external observer is restricted to detect the output of the function $\varphi$ rather than the event itself executed in the net. Pictorially, circles represent the places and rectangles the transitions, while capital letters, written close to the places, represent the output signals of the net. With a slight abuse of notation, let $I\left(p_{i}, t_{j}\right)$ denotes the arc from the place $p_{i}$ to the transition $t_{j}$. Thus, if this arc exists in a model, then $I\left(p_{i}, t_{j}\right)=1$, otherwise $I\left(p_{i}, t_{j}\right)=0$. Similarly, let $O\left(p_{i}, t_{j}\right)$ denotes the arc from the transition $t_{j}$ to the place $p_{i}$. Then, $O\left(p_{i}, t_{j}\right)=1$ means that the arc exists, and $O\left(p_{i}, t_{j}\right)=0$ otherwise. The matrices $B^{-}(i, j):=I\left(p_{i}, t_{j}\right)$ and $B^{+}(i, j):=O\left(p_{i}, t_{j}\right)$ capture the structure of these flow functions. Thus, $B^{-}(i, j)$ is the flow relation from the place $p_{i}$ to the transition $t_{i}$ while $B^{+}(i, j)$ is the flow relation from the transition $t_{j}$ to the place $p_{i}$. Based on these matrices, the incidence matrix is $B:=B^{+}-B^{-}$. Notice that $B(i, j)=0$ is possible while $B^{-}(i, j) \neq 0$ and $B^{+}(i, j) \neq 0$. Thus, $B^{-}(i, j)=B^{+}(i, j)$. This is called a self-loop. The detection of self-loops is useless in the context of DES. Accordingly, this work considers no self-loops in the analyzed OPN.

The pre-set and post-set of $t_{j} \in T$, are $\diamond t_{j}:=\left\{p_{i} \in P: B^{-}(i, j)>0\right\}$ and, respectively $t_{j} \diamond:=\left\{p_{i} \in P: B^{+}(i, j)>0\right\}$. Likewise, for $p_{i} \in P \quad$ are $\diamond p_{i}:=\left\{t_{j} \in T: B^{+}(i, j)>0\right\} \quad$ and $p_{i} \diamond:=\left\{t_{j} \in T: B^{-}(i, j)>0\right\}$. The $\diamond$ operator is extended in a natural way for both sets, of places or of transitions. An OPN is said to be connected if, $\forall u, v \in P \cup T$, exists a path from $u$ to $v$ and from $v$ to $u$, in the sense of the automata theory [7], where the arcs are defined by $B^{-}$and $B^{+}$.

The state, or marking, of an OPN is $M(k) \in\left(\mathbb{N}^{+}\right)^{m}$, with $m=|P|$. The marking represents the number of tokens on each place at time $k$. For convenience, this paper represents $M(k)$ by $M_{k}$, and it uses the notation $M_{k}\left(p_{i}\right), p_{i} \in P$, for representing the number of tokens in $p_{i}$ at time $k$. Moreover, given a marking $M_{k}\left(p_{i}\right)=x$, for $p_{i} \in P$ and $x \in \mathbb{N}^{+}$, it is represented as $M_{k}\left\{\begin{array}{ll}x & p_{i}\end{array}\right\}$. Thus, $M_{k}\left\{2 p_{i}, p_{j}\right\}$ means two tokens in $p_{i}$ and one in $p_{j}$. Notice that curly brackets replace parentheses. The initial marking $M_{0} \in\left(\mathbb{N}^{+}\right)^{m}$ is a primary distribution of tokens in each place, and is used for representing the initial condition of a DES. Thus, $M_{0}\left(p_{i}\right)$ is the initial number of tokens in $p_{i}$. The marking $M_{0}$ may enable the firing of transitions. A transition $t_{i} \in T$ is enabled at $M_{0}$, denoted $\left[M_{0}\right) t_{i}$, if $M_{0}\left(p_{j}\right) \geq B^{-}(i, j), \forall p_{j} \in P$. Given $M_{k}$, the notation $\left[M_{k}\right\rangle$ represents the set of all its enabled transitions. The state equation of an OPN is as follows:

$$
M_{k+1}=M_{k}+B \vec{u}_{k}, y_{k}=\varphi\left(M_{k}\right)
$$

where $M_{k}$ represents the net's state at time $k$; the Parikh vector $\vec{u}_{k}$ represents the firing of one or more transitions enabled at $M_{k}$, and $B$ is the incidence matrix, as defined before. The vector $M_{k+1}$ is the state reached by the net and, $y_{k}$ is the $k$-th system output. Notice that $\varphi\left(M_{k}\right):=\varphi M_{k}$ since $\varphi$ is linear. This fact is used interchangeably where no confusion arises. As with the markings, $y_{k}\{2 A, G\}$ means that the sensor $\mathrm{A}$ is measuring two tokens and $\mathrm{G}$ one token, at the time $k$.

If $\left[M_{k}\right\rangle t_{j}$ and $t_{j}$ is fired, then by using (1) $M_{k+1}=M_{k}+B \vec{t}_{j}=M_{k}+B\left(:, t_{j}\right)$. In this computation, $\vec{u}_{k}=\vec{t}_{j}$ is the Parikh vector with a one in the $j-$ th position and zero anywhere else. This evolution is denoted as $M_{k} \stackrel{t_{i}}{\rightarrow} M_{k+1}$, to emphasize the fact that from $M_{k}, t_{j}$ is 
fired reaching $M_{k+1}$. The reachability set, denoted by $R\left(B, M_{0}\right)$, is recursively defined as the union of all markings reached by the firing of enabled transitions, where by definition $M_{0} \in R\left(B, M_{0}\right)$. A net is safe if $\forall M_{k} \in R\left(B, M_{0}\right)$, it holds that $M_{k}\left(p_{i}\right) \leq 1, \forall p_{i} \in P$, and non-safe otherwise. The compact representation of any marking $M_{k}$ in a safe net is always of the form $M_{k}\left\{p_{i}, \ldots, p_{j}\right\}$ for the set of places marked with a token at $M_{k}$.

A transitions sequence is a string $\sigma=t_{i} t_{j} t_{k} \cdots t_{l}$ such that $M_{0} \stackrel{t_{i}}{\rightarrow} M_{1} \stackrel{t_{j}}{\rightarrow} M_{2} \stackrel{t_{k}}{\rightarrow} \cdots M_{l} \stackrel{t_{l}}{\rightarrow} M_{s}$. The length of $\sigma$, denoted $|\sigma|$, is the number of its transitions. An infinite number of transitions is possible in $\sigma$, in such a case $|\sigma|=\infty$. This trajectory, or sequence, is denoted by $M_{0} \stackrel{\sigma}{\rightarrow} M_{s}$. If $M_{k} \stackrel{\sigma}{\rightarrow} M_{s}$ for some $M_{k}$ and $\sigma$, then the notation $\left[M_{k}\right\rangle \sigma$ means that $M_{k}$ enables the firing of the whole sequence $\sigma$. Formally, the Parikh vector $\vec{\sigma} \in\left(\mathbb{N}^{+}\right)^{n}$, is a map for every transition in the set $T$ to its number of occurrences in $\sigma$. Thus, if $\sigma=t_{i} t_{j} t_{i}$, then $\vec{\sigma}$ is a n-vector, $n=|T|$, with $\vec{\sigma}(i)=2, \vec{\sigma}(j)=1$, and zero anywhere else. As in the automata theory [7], given a sequence $\sigma$ ,$\sigma^{*}$ denotes its Kleen closure that extends in a natural way for sets. The firing language of $\left(B, M_{0}, \varphi\right)$ is

$$
L\left(B, M_{0}\right):=\left(\sigma \in T^{*}: \sigma=t_{i} t_{j} t_{k} \ldots t_{l}\right)
$$

such that $\quad M_{0} \stackrel{t_{i}}{\rightarrow} M_{1} \stackrel{t_{j}}{\rightarrow} M_{2} \stackrel{t_{k}}{\rightarrow} \cdots M_{r} \stackrel{t_{t}}{\rightarrow} M_{s}$. Similarly, the middle-language is $\overline{L\left(B, M_{0}\right)}:=\left(\beta \in T^{*} \mid \exists \alpha, \gamma: \alpha \beta \gamma \in L\left(B, M_{0}\right)\right)$, where $\alpha$ and $\gamma$ could be empty. Clearly, $L\left(B, M_{0}\right) \subseteq \overline{L\left(B, M_{0}\right)}$.

The output word associated to $\sigma \in \overline{L\left(B, M_{0}\right)}$ is $\varphi(\sigma):=\varphi\left(M_{k}\right) \varphi\left(M_{k+1}\right) \ldots \varphi\left(M_{k+r}\right) \varphi\left(M_{k+s}\right)$

whit $M_{k} \stackrel{t_{i}}{\rightarrow} M_{k+1} \stackrel{t_{j}}{\rightarrow} M_{k+2} \stackrel{t_{k}}{\rightarrow} \cdots M_{k+r} \stackrel{\stackrel{t_{l}}{\rightarrow}}{\rightarrow} M_{k+s}$ and $\sigma=t_{i} t_{j} t_{k} \cdots t_{l}$, where $k \geq 0$ and $|\sigma|=s$. Usually, a real DES has a reduced number of sensors, so it is possible that markings $M_{k} \neq M_{k}^{\prime}$ appear alike, i.e. $\varphi M_{k}=\varphi M_{k}^{\prime}$, to an external observer. Therefore, $\left(M_{k}-M_{k}^{\prime}\right) \in \operatorname{ker} \varphi$. Thus, it is possible that for an initial marking $M_{0}$, there may be another one, say $M_{0}^{\prime}$, with $\varphi M_{0}=\varphi M_{0}^{\prime}$. Thus, by using the output information, it may not be direct to determine the initial state, the final state, or even the sequence of transitions executed by a DES. Indeed, by (1), $M_{k+1}=M_{k}+B \vec{u}_{k}$. Then, $\varphi M_{k+1}=\varphi M_{k}+\varphi B \vec{u}_{k}$. The vector $\varphi B \vec{u}_{k}$, denoted by $\varphi_{B}\left(\vec{u}_{k}\right)$, is interpreted as the change, or increment, in the system sensors due to $u_{k}$, whose Parikh vector is $\vec{u}_{k}$. For example, if $\varphi_{B}\left(\vec{u}_{k}\right)=\{-A, B\}$, it means that the firing of $\vec{u}_{k}$ turns off the sensor A, while it turns on the sensor B.

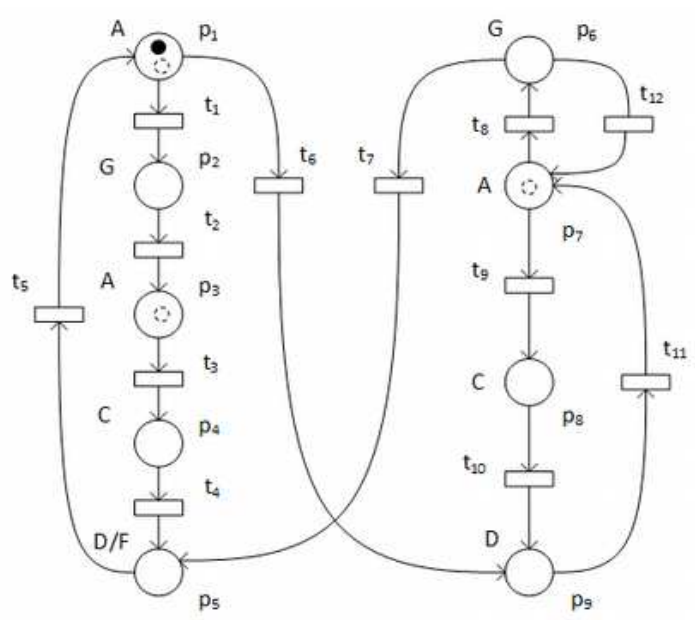

Figure 1. A well-formed OSS.

However, if $\operatorname{ker} \varphi \neq \varnothing$ then it may exist another sequence exists, say $u_{s}$, with $\vec{u}_{s} \neq \vec{u}_{k}$, such that $\varphi_{B}\left(\vec{u}_{s}\right)=\varphi_{B}\left(\vec{u}_{k}\right)$. Notice that it is possible that $\varphi_{B}\left(\vec{t}_{i}\right)=0$, while $B \vec{t}_{i} \neq 0$. Such a transition $t_{i}$ is known as silent. The silent transitions lead to a resilience in the detection of the trajectories as in [14]. However, this work is focused on a constant detection of the trajectories and does not consider silent transitions. More notions of PN's are in [4], [13].

\section{Sequence Detection Analysis}

Roughly speaking, the Sequence Detection problem deals with the determination of the transition sequence executed by an OPN model.

Definition 1. An $O P N\left(B, M_{0}, \varphi\right)$, where $M_{0}$ may be unknown, is Sequence-Detectable (SD) if there exists an integer, $k_{S}<\infty$, such that for any $\sigma \in L\left(B, M_{0}\right)$ with $|\sigma| \geq k_{S}$, where $\sigma=t_{i} t_{j} t_{k} \ldots t_{l}$ and $M_{0} \stackrel{\sigma}{\rightarrow} M_{s}$, the information of the output word $\varphi(\sigma)$ and the structure of $\left(B, M_{0}, \varphi\right)$ allows to uniquely determine $\sigma$. 
Testing the SD is a complex task in the general case, since it may require the examination of all $L\left(B, M_{0}\right)$. Fortunately, efficient algorithms are derived for a set of OPN's called S-Systems [4].

Definition 2. An Output S-System (OSS) is an OPN where $\forall t_{i} \in T:\left(\diamond t_{i}\right)=1=\left(t_{i} \diamond\right)$.

The transitions of an OSS have only one input and one output. The simplicity of the structure confers to the matrix $B$ useful properties for an efficient testing of the SD property. A well-formed OSS is strongly connected (see Pag. 28, Theorem 2.25 in [4]). Additionally, these nets are conservative, in the sense that its total number of tokens remains the same for any evolution (see Pag. 43, Theorem 3.6 in [4]). Where no confusion arises, the analyzed OSS are considered to be well-formed, as in Figure 1.

\subsection{Sequence detectability in safe OSS}

The following example illustrates the concepts developed in this paper, providing intuitive ideas about the sequence detection in an OSS.

Example 1. Consider the well-formed OSS in Figure 1. The net is live and safe since the initial marking puts exactly one token on $p_{1}$. Thus $\sum M_{k}\left(p_{i}\right)=1, \forall p_{i} \in P \quad$ for every marking $M_{k} \in R\left(B, M_{0}\right)$. The sensor of $p_{5}$ is considered to be $D$ and the initial marking $M\left\{p_{1}\right\}$. The $F$ signal for $p_{5}$ and the dashed tokens in $p_{1}, p_{3}$ and $p_{7}$, are considered in further examples. Notice that $\varphi M\left\{p_{1}\right\}=\varphi M\left\{p_{3}\right\}=\varphi M\left\{p_{7}\right\}$, since $p_{1}, p_{3}$, $p_{7}$ have the same sensor. Thus, an external observer is unable to decide the initial condition of the OSS by only using $Y_{0}=\varphi M\left\{p_{1}\right\}$. Moreover, $M\left\{p_{1}\right\}$ enables the firing of both, $t_{1}$ and $t_{6}$. If $t_{6}$ is fired, then an external observer can immediately detect its execution since $\varphi_{B}\left(\vec{t}_{6}\right)=\{-A, D\}$ is different from any other firing. However, if $t_{1}$ is fired then its execution is confused with that of $t_{8}$, i.e. $\varphi_{B}\left(\vec{t}_{1}\right)=\varphi_{B}\left(\vec{t}_{8}\right)$. Moreover, notice that $t_{8}$ is indeed enabled at $M\left\{p_{7}\right\}$. So, by the solely information provided by a single firing, it is impossible to decide which of the transitions, either $t_{1}$ or $t_{8}$, have fired. Besides, it is simple to verify that the firing of $t_{2}$ is confused with that of $t_{12}$, and $t_{3}$ with $t_{9}$, as well. Indeed, $\sigma_{1}=t_{1} t_{2} t_{3} t_{4} t_{5}$ and $\sigma_{2}=t_{8} t_{12} t_{9} t_{10} t_{11}$ are confusing to each other. This is a pair of transition circuits whose firing is confusing, i.e.,

$$
\varphi\left(t_{1} t_{2} t_{3} t_{4} t_{5}\right)=\varphi\left(t_{8} t_{12} t_{9} t_{10} t_{11}\right) \text {. }
$$

The circuits analyzed in the previous example, are closely related to the SD of a safe OSS. In [3], the authors showed that an intersection of vector spaces suffices for testing the property. This work extends this idea and proposes a full condition for testing the property.

Definition 3. Let $\left(B, M_{0}, \varphi\right)$ be a safe OSS, with $T=\left\{t_{1}, \ldots, t_{n}\right\}$ is its set of transitions. The Event-Detectability table $E_{B}$ (Table 1) is a square arrangement of size $[n-1 \times n-1]$, with columns representing the transitions from $t_{1}$ to $t_{n-1}$ and rows representing the transitions from $t_{2}$ to $t_{n}$. The entries of $E_{B}$ are as follows. For $2 \leq i \leq n ; 1 \leq j \leq n-1 ; j<i$ :

Table 1. The Event-Detectability table $E_{B}$.

\begin{tabular}{|c|c|c|c|c|c|c|c|c|c|c|c|}
\hline$t_{2}$ & \{\} & & & & & & & & & & \\
\hline$t_{3}$ & \{\} & \{\} & & & & & & & & & \\
\hline$t_{4}$ & \{\} & \{\} & \{\} & & & & & & & & \\
\hline$t_{5}$ & \{\} & \{\} & \{\} & \{\} & & & & & & & \\
\hline$t_{6}$ & \{\} & \{\} & \{\} & \{\} & \{\} & & & & & & \\
\hline$t_{7}$ & \{\} & \{\} & \{\} & \{\} & \{\} & \{\} & & & & & \\
\hline$t_{8}$ & $\begin{array}{l}\left\{\mathrm{t}_{12}, \mathrm{t}_{2}\right\} \\
\left\{\mathrm{t}_{7}, \mathrm{t}_{2}\right\}\end{array}$ & \{\} & \{\} & \{\} & \{\} & \{\} & \{\} & & & & \\
\hline$t_{9}$ & \{\} & \{\} & $\left\{\mathrm{t}_{10}, \mathrm{t}_{4}\right\}$ & \{\} & \{\} & \{\} & \{\} & \{\} & & & \\
\hline$t_{10}$ & \{\} & \{\} & \{\} & $\left\{\mathrm{t}_{11}, \mathrm{t}_{5}\right\}$ & \{\} & \{\} & \{\} & \{\} & \{\} & & \\
\hline$t_{11}$ & \{\} & \{\} & \{\} & \{\} & $\begin{array}{l}\left\{\mathrm{t}_{12}, \mathrm{t}_{1}\right\} \\
\left\{\mathrm{t}_{12}, \mathrm{t}_{6}\right\} \\
\left\{\mathrm{t}_{8}, \mathrm{t}_{1}\right\} \\
\left\{\mathrm{t}_{8}, \mathrm{t}_{6}\right\}\end{array}$ & \{\} & \{\} & \{\} & \{\} & \{\} & \\
\hline$t_{12}$ & \{\} & $\begin{array}{l}\left\{t_{9}, t_{3}\right\} \\
\left\{t_{8}, t_{3}\right\}\end{array}$ & \{\} & \{\} & \{\} & \{\} & \{\} & \{\} & \{\} & \{\} & \{\} \\
\hline & $t_{1}$ & $t_{2}$ & $t_{3}$ & $\mathrm{t}_{4}$ & $t_{5}$ & $t_{6}$ & $t_{7}$ & $t_{8}$ & $t_{9}$ & $\mathrm{t}_{10}$ & $t_{11}$ \\
\hline
\end{tabular}




$$
\begin{gathered}
\text { if } \varphi_{B}\left(\vec{t}_{i}\right) \neq \varphi_{B}\left(\vec{t}_{j}\right), \\
\text { then } E_{B}\left(t_{i}, t_{j}\right)=\varnothing, \text { otherwise, } \\
E_{B}\left(t_{i}, t_{j}\right)=\cup\left\{t_{u}, t_{v}\right\} \\
\forall t_{u} \in\left(t_{i} \diamond\right) \diamond, \forall t_{v} \in\left(t_{j} \diamond\right) \diamond
\end{gathered}
$$

The elements of $E_{B}$ are pairs in $T \times T$. It is easily noticed that the shape of $E_{B}$ is a kind of lower-triangular. If the firing of $t_{i}$ is different from that of $t_{j}$, then $E_{B}\left(t_{i}, t_{j}\right)=\varnothing$. Otherwise, it is filled with the union of pairs in the Cartesian product $\left(\left(t_{i} \diamond\right) \diamond\right) \times\left(\left(t_{j} \diamond\right) \diamond\right)$. Notice that if the column and row in $E_{B}$ corresponding to $t_{i}$ are empty, i.e. $E_{B}\left(:, t_{i}\right)=\varnothing$ and $E_{B}\left(t_{i},:\right)=\varnothing$, then the firing of $t_{i}$ is different from any other in the net.

Example 2. Consider the Table 1, showing $E_{B}$ of the OSS in Figure 1. Since $\varphi_{B}\left(\vec{t}_{1}\right)=\varphi_{B}\left(\vec{t}_{8}\right)$, the corresponding entry $E_{B}\left(t_{8}, t_{1}\right)$ includes the pairs $\left(t_{12}, t_{2}\right)$ and $\left(t_{7}, t_{2}\right)$. Similarly, $E_{B}\left(t_{9}, t_{3}\right), \quad E_{B}\left(t_{10}, t_{4}\right), \quad E_{B}\left(t_{11}, t_{5}\right)$ and $E_{B}\left(t_{12}, t_{2}\right)$ are non-empty, meaning that its firing is confused to some others. Notice that the entry $E_{B}\left(t_{11}, t_{5}\right)$ includes four pairs, $\left(t_{8}, t_{1}\right),\left(t_{8}, t_{6}\right),\left(t_{12}, t_{1}\right)$ and $\left(t_{12}, t_{6}\right)$, since $\left(t_{11} \diamond\right) \diamond=\left\{t_{8}, t_{12}\right\}$ and $\left(t_{5} \diamond\right) \diamond=\left\{t_{1}, t_{6}\right\}$. On the other hand, the rows and columns corresponding to $t_{6}$ and $t_{7}$ are empty. It means that the firing of any of those transitions could be detected by examining $E_{B}$.

If a column or row in $E_{B}$ is nonempty, then an external observer cannot detect the firing of the corresponding transition by solely considering the information in $E_{B}$. However, the information of more firings of transitions contained in the Sequence-Detectability table could be used.

Definition 4. Let $E_{B}$ be the EventDetectability table of the OSS $\left(B, M_{0}, \varphi\right)$. Consider the Sequence-Detectability table $E_{B}^{s}$ (Table 2) obtained from $E_{B}$ by removing $\left(t_{u}, t_{v}\right)$ if and only if $E_{B}\left(t_{u}, t_{v}\right)=\varnothing$, from any other entry where it appears, say $E_{B}\left(t_{i}, t_{j}\right)$, whenever $t_{i}$ and $t_{j}$ share no place, no input nor output, and recursively removing the new entries that become empty. Formally,

$$
E_{B}^{s}\left(t_{i}, t_{j}\right)=E_{B}\left(t_{i}, t_{j}\right) \backslash\left(t_{u}, t_{v}\right) \text { iff } E_{B}\left(t_{u}, t_{v}\right)=\varnothing
$$

and both, $\diamond t_{i} \cap \diamond t_{j}=\varnothing$ and $t_{i} \diamond \cap t_{j} \diamond=\varnothing$, and recursively, repeat the procedure with the new empty entries that may appear.

As a first step, the definition of $E_{B}^{s}$ removes a pair, from any entry where it appears, if its corresponding entry is already empty. Moreover, if new empty entries appear, the recursive step allows its propagation to the entire table $E_{B}^{s}$. Notice the restrictions $\diamond t_{i} \cap \diamond t_{j}=\varnothing$ and $t_{i} \diamond \cap t_{j} \diamond=\varnothing$ at the first point in the Definition 4. By the one hand, if $\diamond t_{i}=p_{i}=\diamond t_{j}$, then trivially $t_{i}$ is enabled in an OSS if and only if $t_{j}$ is enabled as well. Thus, every time that either $t_{i}$ or $t_{j}$ is fired, the executed sequence, say $\sigma t_{i}$ or $\sigma t_{j}$ becomes ambiguous, since $\varphi_{B}\left(\vec{t}_{i}\right)=\varphi_{B}\left(\vec{t}_{j}\right)^{j}$. Moreover, since the net is a connected OSS, such sequence $\sigma$ always exists (see Pag. 182, Theorem 8.11 in [4]). This directly leads to the non-sequence-detectability of the OSS. On the other hand, if $t_{i} \diamond=p_{j}=t_{j} \diamond$ then any transitions sequence, say $t_{i} \sigma$, enabled by an

Table 2. The Sequence-Detectability table $E_{B}^{S}$.

\begin{tabular}{|c|c|c|c|c|c|c|c|c|c|c|c|c|}
\hline $\mathrm{t}_{2}$ & \{\} & & & & & & & & & & \\
\hline $\mathrm{t}_{3}$ & \{\} & \{\} & & & & & & & & & \\
\hline $\mathrm{t}_{4}$ & \{\} & \{\} & \{\} & & & & & & & & \\
\hline $\mathrm{t}_{5}$ & \{\} & \{\} & \{\} & \{\} & & & & & & & \\
\hline $\mathrm{t}_{6}$ & \{\} & \{\} & \{\} & \{\} & \{\} & & & & & & \\
\hline $\mathrm{t}_{7}$ & \{\} & \{\} & \{\} & \{\} & \{\} & \{\} & & & & & \\
\hline $\mathrm{t}_{8}$ & $\left\{\mathrm{t}_{12}, \mathrm{t}_{2}\right\}$ & \{\} & \{\} & \{\} & \{\} & \{\} & \{\} & & & & \\
\hline $\mathrm{t}_{9}$ & \{\} & \{\} & $\left\{\mathrm{t}_{10}, \mathrm{t}_{4}\right\}$ & \{\} & \{\} & \{\} & \{\} & \{\} & & & \\
\hline $\mathrm{t}_{10}$ & \{\} & \{\} & \{\} & $\left\{\mathrm{t}_{11}, \mathrm{t}_{5}\right\}$ & \{\} & \{\} & \{\} & \{\} & \{\} & & \\
\hline $\mathrm{t}_{11}$ & \{\} & \{\} & \{\} & \{\} & $\left\{\mathrm{t}_{8}, \mathrm{t}_{1}\right\}$ & \{\} & \{\} & \{\} & \{\} & \{\} & \\
\hline $\mathrm{t}_{12}$ & \{\} & $\left\{\mathrm{t}_{9}, \mathrm{t}_{3}\right\}$ & \{\} & \{\} & \{\} & \{\} & \{\} & \{\} & \{\} & \{\} & \{\} \\
\hline & $\mathrm{t}_{1}$ & $\mathrm{t}_{2}$ & $\mathrm{t}_{3}$ & $\mathrm{t}_{4}$ & $\mathrm{t}_{5}$ & $\mathrm{t}_{6}$ & $\mathrm{t}_{7}$ & $\mathrm{t}_{8}$ & $\mathrm{t}_{9}$ & $\mathrm{t}_{10}$ & $\mathrm{t}_{11}$ \\
\hline
\end{tabular}


initial marking $M_{0}\left\{p_{i}\right\}$ for $p_{i}=\diamond t_{i}$, could never be distinguished from $t_{j} \sigma$.

At a first glance, it looks like there is a similarity between the construction method of $E_{B}^{s}$ and minimization of automata method in the MyhillNerode theorem (see Chapter 3 in [7]). A further analysis is planned as future work.

Example 3. Table 2 shows $E_{B}^{s}$ for the OSS in Figure 1. Notice that $\left(t_{7}, t_{2}\right)$ has been removed from $E_{B}^{s}\left(t_{8}, t_{1}\right)$, since $E_{B}^{s}\left(t_{7}, t_{2}\right)=\varnothing$ . Similarly, $\left(t_{8}, t_{3}\right)$ from $E_{B}^{s}\left(t_{12}, t_{2}\right)$, as well as $\left(t_{12}, t_{1}\right),\left(t_{12}, t_{6}\right)$ and $\left(t_{8}, t_{6}\right)$ from $E_{B}^{s}\left(t_{11}, t_{5}\right)$. However, $\left(t_{12}, t_{2}\right)$ cannot be removed from $E_{B}^{s}\left(t_{8}, t_{1}\right)$ since $E_{B}^{s}\left(t_{12}, t_{2}\right) \neq \varnothing$ . But, for removing $\left(t_{9}, t_{3}\right)$ from $E_{B}^{s}\left(t_{12}, t_{2}\right)$ it is required removing $\left(t_{10}, t_{4}\right)$ from $E_{B}^{s}\left(t_{9}, t_{3}\right)$. Though, for removing $\left(t_{10}, t_{4}\right)$, it is required take away $\left(t_{11}, t_{5}\right)$ from $E_{B}^{s}\left(t_{10}, t_{4}\right)$. Finally, for removing $\left(t_{11}, t_{5}\right)$ from $E_{B}^{s}\left(t_{10}, t_{4}\right)$ it is required take out $\left(t_{8}, t_{1}\right)$ from $E_{B}^{s}\left(t_{11}, t_{5}\right)$, which is the cyclic dependency discussed in the Example 1.

Let $\Phi=\left\{t_{i}, t_{j}\right\},\left\{t_{k}, t_{l}\right\}, \cdots,\left\{t_{u}, t_{v}\right\} \quad$ be the sequence of entries as in the previous example.

Based on $\Phi$, it is easily constructed $\sigma=\left(\sigma_{1}, \sigma_{2}\right) \quad$ where $\quad \sigma_{1}=t_{i} t_{k} \cdots t_{u} \quad$ and $\sigma_{2}=t_{k} t_{l} \cdots t_{v}$ are the concatenation of consecutive transitions in the pairs of $\Phi$. By the definition of $E_{B}^{s}, \sigma_{1}$ and $\sigma_{2}$ are allowed to fire since $t_{i} \diamond=\diamond t_{k}, t_{j} \diamond=\diamond t_{l}, t_{u} \diamond=\diamond t_{v}$, etc. Clearly $\varphi\left(\sigma_{1}\right)=\varphi\left(\sigma_{2}\right)$. Moreover, $\sigma_{1}$ and $\sigma_{2}$ are T-invariants (see Pag. 45, Lemma 3.9 in [4]). Let $\Delta E_{B}^{s}$ denote the set of circuits, as $\Phi$ , in $E_{B}^{s}$. Then, the next theorem holds.

Theorem 1. A safe OSS $\left(B, M_{0}, \varphi\right)$ is $S D$ if and only if $\Delta E_{B}^{s}=\varnothing$.

Proof: (Necessity) Supposes that $\left(B, M_{0}, \varphi\right)$ is $S D$ but $\Delta E_{B}^{s} \neq \varnothing$. Let $\Phi \in \Delta E_{B}^{s}$. Without loss of generality, let say that $\sigma=\left(\sigma_{1}, \sigma_{2}\right)$ is constructed from $\Phi$ as before, and that $\sigma_{1}=t_{i} t_{k} t_{u} \cdots t_{w} t_{y}$ and $\sigma_{2}=t_{j} t_{l} t_{v} \cdots t_{x} t_{z}$. Thus, in $E_{B}^{s}$ must exist $E_{B}^{s}\left(t_{i}, t_{j}\right), E_{B}^{s}\left(t_{k}, t_{l}\right)$, $E_{B}^{s}\left(t_{u}, t_{v}\right), \quad \ldots \quad, \quad E_{B}^{s}\left(t_{w}, t_{x}\right), \quad E_{B}^{s}\left(t_{y}, t_{z}\right)$, $E_{B}^{s}\left(t_{i}, t_{j}\right) \quad$ such that $\left(t_{k}, t_{l}\right) \in E_{B}^{s}\left(t_{i}, t_{j}\right)$, $\left(t_{u}, t_{v}\right) \in E_{B}^{s}\left(t_{k}, t_{l}\right), \ldots, \quad\left(t_{y}, t_{z}\right) \in E_{B}^{s}\left(t_{w}, t_{x}\right)$, and $\left(t_{i}, t_{j}\right) \in E_{B}^{s}\left(t_{y}, t_{z}\right)$. By Definition 4, it holds that $\varphi_{B}\left(\vec{t}_{i}\right)=\varphi_{B}\left(\vec{t}_{j}\right), \varphi_{B}\left(\vec{t}_{k}\right)=\varphi_{B}\left(\vec{t}_{l}\right)$, $\varphi_{B}\left(\vec{t}_{u}\right)=\varphi_{B}\left(\vec{t}_{v}\right), \quad \ldots, \quad \varphi_{B}\left(\vec{t}_{w}\right)=\varphi_{B}\left(\vec{t}_{x}\right)$, $\varphi_{B}\left(\vec{t}_{y}\right)=\varphi_{B}\left(\vec{t}_{z}\right)$. Moreover, $t_{i} \diamond=\diamond t_{k}$, $t_{j} \diamond=\diamond t_{l}, \quad t_{k} \diamond=\diamond t_{u}, \quad t_{l} \diamond=\diamond t_{v}, \quad \ldots$, $t_{w} \diamond=\diamond t_{y}, \quad t_{x} \diamond=\diamond t_{z}, \quad t_{y} \diamond=\diamond t_{i}$, and $t_{z} \diamond=\diamond t_{j}$. Let $\left\{p_{i}\right\}=\diamond t_{i}$ and $\left\{p_{j}\right\}=\diamond t_{j}$. Since $\left(B, M_{0}, \varphi\right)$ is live, it is place live, as well (see Pag. 26, Proposition 2.17 in [4]). Then, clearly $M\left\{p_{i}\right\}$ and $M^{\prime}\left\{p_{j}\right\}$ are valid markings of the net. Thus, $\sigma_{1}, \sigma_{2} \in \overline{L\left(B, M_{0}\right)}$ where $\sigma_{1}=t_{i} t_{k} t_{u} \ldots t_{w} t_{v}$ and $\sigma_{2}=t_{j} t_{l} t_{v} \ldots t_{x} t_{z}$, since $\left[M\left\{p_{i}\right\}\right\} \sigma_{1}$ and $\left[M^{\prime}\left\{p_{j}\right\}\right) \sigma_{2}$. Let

$$
\begin{aligned}
& M\left\{p_{i}\right\} \stackrel{t_{i}}{\rightarrow} M\left\{p_{k}\right\} \stackrel{t_{k}}{\rightarrow} M\left\{p_{u}\right\}^{t_{u}} \rightarrow \cdots \\
& \cdots M\left\{p_{w}\right\} \stackrel{t_{w}}{\rightarrow} M\left\{p_{y}\right\} \stackrel{t_{v}}{\rightarrow} M\left\{p_{i}\right\}
\end{aligned}
$$

and

$$
\begin{aligned}
& M^{\prime}\left\{p_{j}\right\}^{t_{j}} \rightarrow M^{\prime}\left\{p_{l}\right\}^{t_{l}} \rightarrow M^{\prime}\left\{p_{v}\right\} \stackrel{t_{v}}{\rightarrow} \cdots \\
& \cdots M^{\prime}\left\{p_{x}\right\}^{t_{x}} \rightarrow M^{\prime}\left\{p_{z}\right\} \stackrel{t_{z}}{\rightarrow} M^{\prime}\left\{p_{j}\right\}
\end{aligned}
$$

be the two sequences of markings due to $\sigma_{1}$ and $\sigma_{2}, \quad$ respectively. Clearly $\varphi M\left\{p_{i}\right\}=\varphi M^{\prime}\left\{p_{j}\right\}, \quad \varphi M\left\{p_{k}\right\}=\varphi M^{\prime}\left\{p_{l}\right\}$, $\varphi M\left\{p_{u}\right\}=\varphi M^{\prime}\left\{p_{v}\right\}, \ldots, \varphi M^{\prime}\left\{p_{w}\right\}=\varphi M^{\prime}\left\{p_{x}\right\}$, $\varphi M\left\{p_{y}\right\}=\varphi M^{\prime}\left\{p_{z}\right\}$, Thus, $\varphi\left(\sigma_{1}\right)=\varphi\left(\sigma_{2}\right)$. Moreover, since $\sigma_{1}$ and $\sigma_{2}$ are circuits, then $\sigma_{1}^{*}, \sigma_{2}^{*} \in \overline{L\left(B, M_{0}\right)}$ (see Pag. 45, Lemma 3.9 in [4]). Thus,

$$
\varphi\left(\sigma_{1}^{*}\right)=\varphi\left(\sigma_{1}\right) \varphi\left(\sigma_{1}\right) \cdots=\varphi\left(\sigma_{2}\right) \varphi\left(\sigma_{2}\right) \cdots=\varphi\left(\sigma_{2}^{*}\right)
$$

which contradicts the net is $S D$.

(Sufficiency) Suppose that $\Delta E_{B}^{s}=\varnothing$ but on the contrary, the net is non-SD. Then, there exist at least two sequences of infinite length, say $\tau_{1}=t_{i} \cdots t_{k} t_{u} \cdots t_{w} t_{y} \cdots$ and $\tau_{2}=t_{j} t_{l} t_{v} \cdots t_{x} t_{z} \cdots$ where $\tau_{1}, \tau_{2} \in \overline{L\left(B, M_{0}\right)}$, such that $\tau_{1} \neq \tau_{2}$, and $\varphi\left(\tau_{1}\right)=\varphi\left(\tau_{2}\right)$. Since $\left(B, M_{0}, \varphi\right)$ is safe, then any initial marking that puts one token on any place of the net, is a home marking (see Pag. 169, Proposition 8.2 in [4]). This turns the selection of $\tau_{1}$ and $\tau_{2}$ independent of any initial marking. It immediately follows that $\varphi_{B}\left(\vec{t}_{i}\right)=\varphi_{B}\left(\vec{t}_{j}\right), \ldots, \varphi_{B}\left(\vec{t}_{k}\right)=\varphi_{B}\left(\vec{t}_{l}\right)$, $\varphi_{B}\left(\vec{t}_{u}\right)=\varphi_{B}\left(\vec{t}_{v}\right), \ldots, \varphi_{B}\left(\vec{t}_{w}\right)=\varphi_{B}\left(\vec{t}_{x}\right)$, $\varphi_{B}\left(\vec{t}_{y}\right)=\varphi_{B}\left(\vec{t}_{z}\right), \ldots$, and so on, since $\varphi\left(\tau_{1}\right)=\varphi\left(\tau_{2}\right)$. Now, consider the sequence $\Phi=\left(t_{i}, t_{j}\right), \ldots,\left(t_{k}, t_{l}\right),\left(t_{u}, t_{v}\right), \ldots,\left(t_{w}, t_{x}\right)$ , $\left(t_{y}, t_{z}\right),\left(t_{k}, t_{l}\right) \ldots$, etc., formed by pairing the transitions that are confused to each other 
in $\tau_{1}$ and $\tau_{2}$, correspondingly. Since $\Phi$ is of infinite length, then at least one of its elements must appear twice, as $|T \times T|<\infty$. Let say, without loss of generality, that the first element repeating in $\Phi$ is $\left\{t_{k}, t_{l}\right\}$. Notice that, in $\tau_{1}$, the firing of $t_{k}$ enables that of $t_{u}$, since the net is safe. Also, in $\tau_{2}$, the firing of $t_{l}$ enables that of $t_{v}$. Indeed, this holds for any two consecutive transitions in $\tau_{1}$ and in $\tau_{2}$. Therefore, in $\tau_{1}$ it holds that $t_{u} \in\left(t_{k} \diamond\right) \diamond, \ldots$, $t_{y} \in\left(t_{w} \diamond\right) \diamond, \quad t_{k} \in\left(t_{y} \diamond\right) \diamond$, and so on. Similarly, in $\tau_{2}$ it holds that $t_{v} \in\left(t_{l} \diamond\right) \diamond, \ldots$, $t_{z} \in\left(t_{x} \diamond\right) \diamond, t_{l} \in\left(t_{z} \diamond\right) \diamond$, and so on. Thus, in the construction of $E_{B}$, it immediately follows that, at least $\left(t_{u}, t_{v}\right) \in E_{B}\left(t_{k}, t_{l}\right), \ldots$, $\left(t_{y}, t_{z}\right) \in E_{B}\left(t_{w}, t_{x}\right), \quad\left(t_{k}, t_{l}\right) \in E_{B}\left(t_{y}, t_{z}\right), \ldots$, and so on. Notice that the last element in this sequence emphasizes the fact that the pair $\left(t_{k}, t_{l}\right)$ belongs to the entry $E_{B}\left(t_{y}, t_{z}\right)$. This is consistent with the previous assumption in the sense that $\left(t_{k}, t_{l}\right)$ is the first element appeared twice in $\Phi$. Now, consider the construction of $E_{B}^{s}$. Clearly, the recursion step cannot remove $\left(t_{u}, t_{v}\right)$ from $E_{B}^{s}\left(t_{k}, t_{l}\right)$ nor $\left(t_{y}, t_{z}\right)$ from $E_{B}^{s}\left(t_{w}, t_{x}\right)$. Moreover, the same apply to any of the intermediate elements in the above sequence of elements of $E_{B}$. Indeed, it finally holds that $\left(t_{k}, t_{l}\right)$ cannot be removed from $E_{B}^{s}\left(t_{y}, t_{z}\right)$. But, $\quad\left(t_{y}, t_{z}\right) \in E_{B}^{s}\left(t_{w}, t_{x}\right)$. This conforms a cyclic dependency, and $\Delta E_{B}^{s} \neq \varnothing$.

The next proposition goes further and shows that any non-empty entry in $E_{B}^{S}$ leads to a circuit.

Proposition 1. Let $E_{B}^{s}$ be the SD table of the OSS $\left(B, M_{0}, \varphi\right)$. Then, for any nonempty entry $E_{B}^{s}\left(t_{g}, t_{h}\right)$ there exist a pair of circuits, say $\sigma_{g}$ and $\sigma_{h}$, such that $\varphi\left(\sigma_{g}\right)=\varphi\left(\sigma_{h}\right)$.

Proof: Let $E_{B}^{s}\left(t_{g}, t_{h}\right) \neq \varnothing$ be any non-empty entry in $E_{B}^{s}$. Then, by construction, at least $E_{B}^{s}\left(t_{g}, t_{h}\right) \supseteq\left(t_{i}, t_{j}\right)$ for some $t_{i}, t_{j}$ that fulfil $\varphi_{B}\left(\vec{t}_{g}\right)=\varphi_{B}\left(\vec{t}_{h}\right)$ and $\varphi_{B}\left(\vec{t}_{i}\right)=\varphi_{B}\left(\vec{t}_{j}\right)$. By supposition both, $\diamond t_{g} \neq \diamond t_{h}$ and $t_{g} \diamond \neq t_{h} \diamond$. Then, necessarily $E_{B}^{s}\left(t_{i}, t_{j}\right) \neq \varnothing$. Otherwise, if $E_{B}^{s}\left(t_{i}, t_{j}\right)=\varnothing$, then the recursive step in Definition 4 must delete $\left(t_{i}, t_{j}\right)$ from $E_{B}^{s}\left(t_{g}, t_{h}\right)$, which contradicts the fact that $E_{B}^{s}\left(t_{g}, t_{h}\right) \neq \varnothing$. Thus, let $E_{B}^{s}\left(t_{i}, t_{j}\right) \supseteq\left(t_{k}, t_{l}\right)$, for $t_{k}, t_{l} \in T$. Then, $\varphi_{B}\left(\vec{t}_{k}\right)=\varphi_{B}\left(\vec{t}_{l}\right)$. Again, since $\diamond t_{i} \neq \diamond t_{j}$ and $t_{i} \diamond \neq t_{j} \diamond$, then it requires that $E_{B}^{s}\left(t_{k}, t_{l}\right) \neq \varnothing$. Let say that $E_{B}^{s}\left(t_{k}, t_{l}\right) \supseteq\left(t_{u}, t_{v}\right)$, with $\varphi_{B}\left(\vec{t}_{u}\right)=\varphi_{B}\left(\vec{t}_{v}\right)$. Otherwise, it easily leads a contradiction as before. Moreover, it should be that $E_{B}^{s}\left(t_{u}, t_{v}\right) \neq \varnothing$. By using this reasoning, without loss of generality, let say that the sequence of entries continues as $E_{B}^{s}\left(t_{u}, t_{v}\right), \ldots$

$E_{B}^{s}\left(t_{w}, t_{x}\right), \quad E_{B}^{s}\left(t_{y}, t_{z}\right), \ldots, \quad \ldots$ etc.

Furthermore, since the net is connected, for every $t_{i}$ there is always other $t_{j} \in\left(t_{i} \diamond\right) \diamond$. Thus, this sequence of entries in $E_{B}^{s}$ shall continue, and since the number of the entries in this table is finite, then at least one of its elements should be repeated. Let say that $\left(t_{k}, t_{l}\right)$ is the first pair that repeating twice, i.e., $E_{B}^{s}\left(t_{y}, t_{z}\right) \supseteq\left(t_{k}, t_{l}\right)$. Clearly, this selection does not cause loss of generality in the result. Now, let $\sigma_{g}=t_{k} t_{u} \cdots t_{w} t_{y}$ and $\sigma_{h}=t_{l} t_{v} \cdots t_{x} t_{z}$ be two transition sequences built from this sequence of entries in $E_{B}^{s}$. Then, clearly $\varphi_{B}\left(\vec{t}_{k}\right)=\varphi_{B}\left(\vec{t}_{l}\right), \quad \varphi_{B}\left(\vec{t}_{u}\right)=\varphi_{B}\left(\vec{t}_{v}\right), \quad \ldots \quad$, $\varphi_{B}\left(\vec{t}_{w}\right)=\varphi_{B}\left(\vec{t}_{x}\right)$, and $\varphi_{B}\left(\vec{t}_{y}\right)=\varphi_{B}\left(\vec{t}_{z}\right)$, i.e., $\varphi\left(\sigma_{g}\right)=\varphi\left(\sigma_{h}\right)$. Thus, the sequence $E_{B}^{s}\left(t_{k}, t_{l}\right)$, $E_{B}^{s}\left(t_{u}, t_{v}\right), \cdots, \quad E_{B}^{s}\left(t_{w}, t_{x}\right), \quad E_{B}^{s}\left(t_{y}, t_{z}\right)$, $E_{B}^{s}\left(t_{k}, t_{l}\right)$ is a circular chain of entries in $E_{B}^{s}$ where, by Definition 4 it holds that $t_{k} \diamond=\diamond t_{u}$, $t_{l} \diamond=\diamond t_{v}, \ldots, t_{w} \diamond=\diamond t_{y}$ and $t_{x} \diamond=\diamond t_{z}$. Hence, $\sigma_{g}$ and $\sigma_{h}$ are circuits of transitions in $\left(B, M_{0}, \varphi\right)$ as required.

From Theorem 3 and Proposition 1, the following corollary holds.

Corollary 1. Let $\left(B, M_{0}, \varphi\right)$ be a safe OSS. Then, $\Delta E_{B}^{s}=\varnothing$ iff every entry in $E_{B}^{s}$ is empty.

\subsection{Sequence detectability in non-safe OSS}

Suppose that the net in Figure 1 is able to hold more than one token. Thus, the marking $M_{0}\left\{p_{1}, p_{7}\right\}$ is a suitable initial condition of the OSS. It is easy to verify that $\left[M_{0}\right) t_{1}$, $\left[M_{0}\right) t_{8}$ and, as discussed earlier, $\varphi_{B}\left(\vec{t}_{1}\right)=\varphi_{B}\left(\vec{t}_{8}\right)$. Now, suppose that the entire sequence $\sigma=t_{1} t_{2} t_{3} t_{4} t_{5}$ is fired. Notice that $\sigma$ is enabled at $M_{0}\left\{p_{1}, p_{7}\right\}$. Clearly, the marking reached by the firing of $\sigma$ is a new $M_{0}\left\{p_{1}, p_{7}\right\}$, since $B \vec{\sigma}=\overrightarrow{0}$. Thus, the firing of $\sigma t_{1}$ confuses with that of $\sigma t_{8}$, i.e., 
$\varphi\left(\sigma t_{1}\right)=\varphi(\sigma) \varphi_{B}\left(\vec{t}_{1}\right)=\varphi(\sigma) \varphi_{B}\left(\vec{t}_{8}\right)=\varphi\left(\sigma t_{8}\right)$.

Since $\sigma$ may execute forever, then

$$
\begin{aligned}
& \varphi\left(\sigma \cdots \sigma t_{1} \cdots\right)=\varphi(\sigma) \cdots \varphi(\sigma) \varphi_{B}\left(\vec{t}_{1}\right) \cdots= \\
& =\varphi(\sigma) \cdots \varphi(\sigma) \varphi_{B}\left(\vec{t}_{8}\right) \cdots=\varphi\left(\sigma \cdots \sigma t_{8} \cdots\right)
\end{aligned} .
$$

It directly implies that the net is not SD. Based in this intuitive idea, the next theorem characterizes the SD in a non-safe OSS.

Theorem 2. Let $\left(B, M_{0}, \varphi\right)$ be a non-safe OSS. Then, the net is $S D$ if and only if $E_{B}$ is empty.

Proof: (Necessity) Supposes that $\left(B, M_{0}, \varphi\right)$ is $S D$ but $E_{B}$ is non-empty. Then, there exist, at least, a pair of transitions, say $t_{i}, t_{j} \in T$, such that $\varphi_{B}\left(\vec{t}_{i}\right)=\varphi_{B}\left(\vec{t}_{j}\right)$. Since the OSS is live, then it is also place-live (see Pag. 26, Proposition 2.17 in [4]). Moreover, since the net is non-safe, by using an enough number of tokens, it is possible to construct the marking $M_{i j} \geq M\left\{p_{i}\right\}+M\left\{p_{j}\right\}$, for $p_{i} \in \diamond t_{i}$ and $p_{j} \in \diamond t_{j}$. Notice that $\exists \sigma \in \overline{L\left(B, M_{0}\right)}$ such that $\quad M_{0} \stackrel{\sigma}{\rightarrow} M_{i j} \quad$ and accordingly $M_{i j} \in R\left(B, M_{0}\right)$. It is clear that $\left[M_{i j}\right) t_{i}$, and $\left[M_{i j}\right] t_{j}$. Thus, it is impossible to distinguish the execution of $\sigma t_{i}$ from $\sigma t_{j}$, since $\varphi\left(\sigma t_{i}\right)=\varphi(\sigma) \varphi_{B}\left(\vec{t}_{i}\right)=\varphi(\sigma) \varphi_{B}\left(\vec{t}_{j}\right)=\varphi\left(\sigma t_{j}\right)$,

as by assumption it holds that $\varphi_{B}\left(\vec{t}_{i}\right)=\varphi_{B}\left(\vec{t}_{j}\right)$. Since every marking in a well-formed OSS is a home marking (see Pag. 169, Proposition 8.2 in [4]), then for each $\sigma t_{i}$ and $\sigma t_{j}$, it should exist other sequences, say $\gamma_{1}, \gamma_{2} \in \overline{L\left(B, M_{0}\right)}$, such that both $\sigma t_{i} \gamma_{1}$ and $\sigma t_{j} \gamma_{2}$ reach $M_{i j}$ anew. Hence, there is a confusion again about the firing of $\sigma t_{i} \gamma_{1} t_{i}$ and $\sigma t_{j} \gamma_{2} t_{j}$, i.e.,

$$
\begin{aligned}
\varphi\left(\sigma t_{i} \gamma_{1} t_{i}\right) & =\varphi\left(\sigma t_{i} \gamma_{1}\right) \varphi_{B}\left(t_{i}\right)= \\
& =\varphi\left(\sigma t_{i} \gamma_{1}\right) \varphi_{B}\left(t_{j}\right)=\varphi\left(\sigma t_{j} \gamma_{2} t_{j}\right) .
\end{aligned}
$$

Applying the same reasoning, it easily leads to the construction of a pair of transition sequences of infinite length that confuses to each other, which directly contradicts the SD of the net.

(Sufficiency) Suppose that $E_{B}$ is empty. Then, $\varphi_{B}\left(\vec{t}_{i}\right) \neq \varphi_{B}\left(\vec{t}_{j}\right)$ for every pair of transitions $t_{i}, t_{j} \in T: t_{i} \neq t_{j}$. Then, the firing of every transition in the system is distinguished from each other. Accordingly, the transition sequence executed by the net from any $M_{0}$, is determined at every execution step by an inspection of $E_{B}$.
The next section uses the results of the sequence detectability for sketching a solution of the observability problem in OSS models.

\section{Observability Analysis}

The observability is a property that allows discovering the state of a DES. This work uses a quite intuitive definition in the OPN field [3].

Definition 5. An $O P N\left(B, M_{0}, \varphi\right)$, where $M_{0}$ may be unknown, is Observable if there exists an integer $k<\infty$, such that for any $\sigma \in L\left(B, M_{0}\right)$ with $(\sigma) \geq k$, where $\sigma=t_{i} t_{j} t_{k} \ldots t_{l}$ and $\quad M_{0} \stackrel{t_{i}}{\rightarrow} M_{1} \stackrel{t_{i}}{\rightarrow} M_{2} \stackrel{t_{k}}{\rightarrow} \cdots M_{r} \stackrel{t_{i}}{\rightarrow} M_{s}$, the information of the output word $\varphi(\sigma)$ and the structure of $\left(B, M_{0}, \varphi\right)$, allows to uniquely determine the markings $M_{0}$ and $M_{s}$.

Based on (1), this work proposes the FiringVector-Detectability and Marking-Detectability properties for testing the observability.

Definition 6. An $O P N\left(B, M_{0}, \varphi\right)$, where $M_{0}$ may be unknown, is Firing-Vector-Detectable (FVD) if there exists an integer $k_{F}<\infty$ such that $\forall \sigma \in L\left(B, M_{0}\right):|\sigma| \geq k_{F}, \quad \sigma=t_{i} t_{j} t_{k} \ldots t_{l}$ with $M_{0} \stackrel{t_{i}}{\rightarrow} M_{1} \rightarrow{ }_{t_{i}}^{\rightarrow} M_{2} \stackrel{t_{k}}{\rightarrow} \cdots M_{r} \stackrel{\stackrel{t_{i}}{\rightarrow}}{\rightarrow} M_{s}$, the output word $\varphi(\sigma)$ and the structure $\left(B, M_{0}, \varphi\right)$, allows to uniquely determine the Parikh vector $\vec{\sigma}$.

Definition 7. An $O P N\left(B, M_{0}, \varphi\right)$, where $M_{0}$ may be unknown, is Marking-Detectable (MD) if there exists an integer $k_{M}<\infty$, such that $\forall \sigma \in L\left(B, M_{0}\right):(\sigma) \geq k_{M}, \quad \sigma=t_{i} t_{j} t_{k} \ldots t_{l}$ with $M_{0} \stackrel{t_{i}}{\rightarrow} M_{1} \stackrel{t_{i}}{\rightarrow} M_{2} \stackrel{t_{k}}{\rightarrow} \cdots M_{r} \stackrel{t_{l}}{\rightarrow} M_{s}$, the output word $\varphi(\sigma)$ and the structure of $\left(B, M_{0}, \varphi\right)$, allows to uniquely determine $M_{k}$ reached by $\sigma$.

By the FVD and MD, next proposition is direct.

Proposition 2. An $O P N\left(B, M_{0}, \varphi\right)$ that is $F V D$ and $M D$ is Observable.

Proof: Let $\left(B, M_{0}, \varphi\right)$ be FVD, with constant $k_{F}$, and $M D$, with constant $k_{M}$. Let $k=\max \left\{k_{F}, k_{M}\right\} \quad$ and let $\sigma \in L\left(B, M_{0}\right):|\sigma| \geq k$. Thus, $\vec{\sigma}$ and $M_{k}$, reached by the firing of $\sigma$, are known, as well as $M_{k}, \vec{\sigma}$ and $B$.

Hence, the initial marking is computed as $M_{0}=M_{k}-B \vec{\sigma}$ by using the state equation (1).

The next subsection uses the SD for testing the FVD and MD of an OSS. 


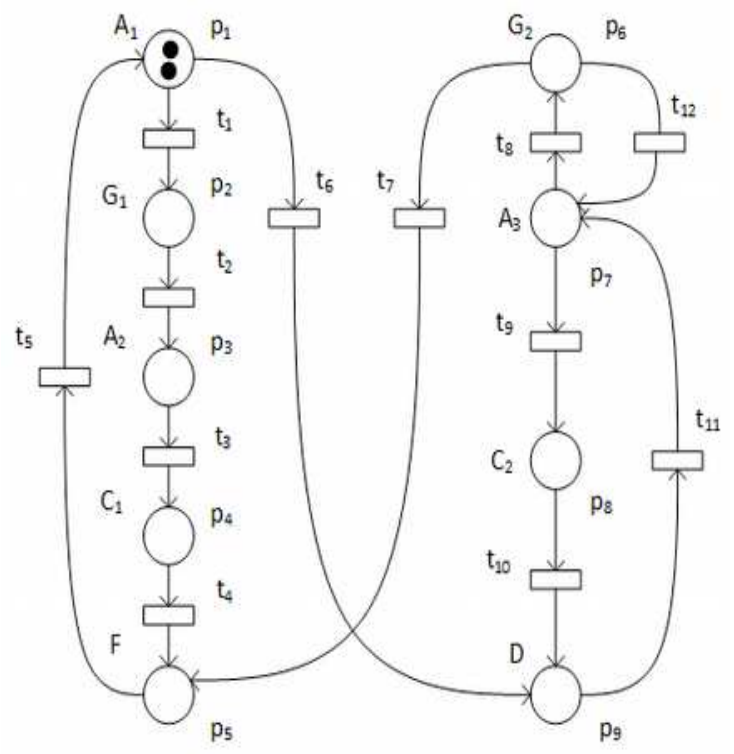

Figure 2. Extra token in $p_{1}$ and the new sensor.

\subsection{Observability in safe OSS}

Clearly, the determination of the Parikh vector from the transition sequence is direct. The next proposition shows that the current marking is also computed easily when the OSS is safe.

Proposition 3. A well-formed and safe OSS $\left(B, M_{0}, \varphi\right)$ that is $S D$, is also $M D$.

Proof: Supposes that $\left(B, M_{0}, \varphi\right)$ is $S D$, with convergence constant $k_{s}$ and let $\sigma \in L\left(B, M_{0}\right)$ such that $|\sigma| \geq k_{s}$. Then $\sigma$ is known. Let say that $\sigma=\alpha t_{j}$ for some $\alpha \in L\left(B, M_{0}\right)$ and $t_{j} \in T$. Without loss of generality, let say that $t_{j} \diamond=\left\{p_{j}\right\}$. Since the net is safe, the marking reached by the firing of $\sigma=\alpha t_{i}$ is clearly $M\left\{p_{j}\right\}$.

Thus, the following corollary is direct and stablishes the observability of a safe OSS.

Corollary 2. If a well-formed and safe OSS is $S D$, then it is observable.

The following example illustrates the result.

Example 4. Consider again the OSS in Figure 1. Suppose that the sensor of $p_{5}$ has been changed from $D$ to $F$. By this change, the firings of $t_{4}$ and $t_{5}$ are now different from any other firing in the net. Thus, $E_{B}^{s}\left(t_{10}, t_{4}\right)$ and $E_{B}^{s}\left(t_{11}, t_{5}\right)$ in the Table 2 must be empty. Therefore, $E_{B}^{s}\left(t_{9}, t_{3}\right)$ becomes empty and hence $E_{B}^{s}\left(t_{12}, t_{2}\right)$, as well. Finally, $E_{B}^{s}\left(t_{8}, t_{1}\right)$ ends up empty and accordingly, $\Delta E_{B}^{s}=\varnothing$. So, the net is now SD.

Notice that the initial marking $M\left\{p_{1}\right\}$ enables the firing of $t_{1}$ as well as that of $t_{6}$. Suppose that $t_{1}$ is fired. Then, an external observer is only able to compute $\sigma_{1}=t_{1}$ and $\sigma_{2}=t_{8}$ as possible executed sequences at $k=1$, since $\varphi_{B}\left(t_{1}\right)=\varphi_{B}\left(t_{8}\right)$. Thus, the possible reached markings are $M\left\{t_{1} \diamond\right\}=M\left\{p_{2}\right\} \quad$ and $M\left\{t_{8} \diamond\right\}=M\left\{p_{6}\right\}$, respectively. Now, suppose that $t_{2}$ is fired. Since $\varphi_{B}\left(t_{2}\right)=\varphi_{B}\left(t_{12}\right)$, then the possible executed sequences, at $k=2$, are $\sigma_{1}=t_{1} t_{2}$ and $\sigma_{2}=t_{8} t_{12}$. Accordingly, the probable reached markings are $M\left\{t_{2} \diamond\right\}=M\left\{p_{3}\right\}$ and $M\left\{t_{12} \diamond\right\}=M\left\{p_{7}\right\}$, correspondingly. It is easy to verify that for $k=3$, the possible executed sequences are $\sigma_{1}=t_{1} t_{2} t_{3}$ and $\sigma_{2}=t_{8} t_{12} t_{9}$, by assuming that $t_{3}$ is fired. The probable reached markings are $M\left\{t_{3} \diamond\right\}=M\left\{p_{4}\right\} \quad$ and $M\left\{t_{9} \diamond\right\}=M\left\{p_{8}\right\}$, respectively. However, at $k=4$ once $t_{4}$ is fired, the sequence $\sigma_{1}=t_{1} t_{2} t_{3} t_{4}$ is distinguished from $\sigma_{2}=t_{8} t_{12} t_{9} t_{10}$. Thus, the observer knows that the sequence executed by the net is $\sigma_{1}$ and it is able to compute its Parikh vector $\vec{\sigma}_{1}$. Moreover, the marking reached by the firing of $\sigma_{1}$ is $M\left\{t_{4} \diamond\right\}=M\left\{p_{5}\right\}$. Finally, the initial marking is $M_{0}=M\left\{p_{5}\right\}-B \vec{\sigma}_{1}=M\left\{p_{1}\right\}$.

\subsection{Observability in non-safe OSS}

The Theorem 2 states that the detection of the transition sequence in a safe OSS allows for detecting its marking. However, it is not the case in non-safe OSS, as detailed in the next example.

Example 5. Consider the markings $M_{0}\left\{p_{1}, p_{3}\right\}, M_{0}^{\prime}\left\{p_{1}, p_{7}\right\}$ and $M_{0}^{\prime \prime}\left\{2 p_{1}\right\}$, as possible initial conditions of the OSS in Figure 1. Those markings are valid since the net is supposed to be non-safe in this case. Clearly, $\varphi M_{0}\left\{p_{1}, p_{3}\right\}=\varphi M_{0}^{\prime}\left\{p_{1}, p_{7}\right\}=\varphi M_{0}^{\prime \prime}\left\{2 p_{1}\right\}$.

Let $\sigma=t_{1} t_{2} t_{3} t_{4} t_{5}$. It is easy verified that $\left[M_{0}\left\{p_{1}, p_{3}\right\}\right\} \sigma$ and consequently $\sigma \in L\left(B, M_{0}\right)$. Notice that $B \vec{\sigma}=\overrightarrow{0}$. Thus, the marking reached at every firing of $\sigma^{*}=\left(t_{1} t_{2} t_{3} t_{4} t_{5}\right)\left(t_{1} t_{2} t_{3} t_{4} t_{5}\right) \cdots$, is ambiguous, since always will exist at least three possible reached markings, either $M_{0}\left\{p_{1}, p_{3}\right\}$, $M_{0}^{\prime}\left\{p_{1}, p_{7}\right\}$ or $M_{0}^{\prime \prime}\left\{2 p_{1}\right\}$. 
Based on the previous idea, the next theorem provides characterizes the MD in non-safe OSS.

Theorem 3. A non-safe OSS $\left(B, M_{0}, \varphi\right)$ is $M D$ if and only if $\operatorname{ker} \varphi=\varnothing$.

Proof: (Necessity) Suppose that $\left(B, M_{0}, \varphi\right)$ is $M D$ but, on the contrary, $\operatorname{ker} \varphi \neq \varnothing$. Then, there exist at least, a linear combination of the columns in $\varphi$ such that $\alpha_{i} \varphi(:, i)+\ldots+\alpha_{j} \varphi(:, j)+\ldots$
$\quad \ldots+\alpha_{k} \varphi(:, k)+\ldots+\alpha_{l} \varphi(:, l)=\overrightarrow{0}$,

where not all the $\alpha^{\prime} s$ coefficients are zero. Since by definition $\varphi$ is non-negative, it must exist a re-arrangement of this linear combination where the elements at both sides of the equality have positive signs. Without loss of generality let

$$
\begin{aligned}
& \alpha_{i} \varphi(:, i)+\ldots+\alpha_{j} \varphi(:, j)= \\
& \quad=\alpha_{k} \varphi(:, k)+\ldots+\alpha_{l} \varphi(:, l)
\end{aligned}
$$

be such an arrangement. Then, by using an enough number of tokens, it is possible the construction of markings $M_{0}\left\{\alpha_{i} p_{i}+\ldots+\alpha_{j} p_{j}\right\}$ and $M_{0}^{\prime}\left\{\alpha_{k} p_{k}+\ldots+\alpha_{l} p_{l}\right\}$ as initial conditions of the net. It is easy to note that

$$
\begin{aligned}
\varphi\left(2 M_{0}\right. & \left.\left\{\alpha_{i} p_{i}+\ldots+\alpha_{j} p_{j}\right\}\right)= \\
& =\varphi\left(M_{0}\left\{\alpha_{i} p_{i}+\ldots+\alpha_{j} p_{j}\right\}+,\right. \\
& \left.+M_{0}^{\prime}\left\{\alpha_{k} p_{k}+\ldots+\alpha_{l} p_{l}\right\}\right)
\end{aligned}
$$

i.e., these markings are confusing to each other. Because the net is well-formed then, it is connected and covered by circuits (see Pag. 106, Theorem 5.18 in [4]). Thus, it must exist a circuit of minimum size including the set of places $\left\{p_{i}, \ldots, p_{j}\right\}$ which are marked at $M_{0}\left\{\alpha_{i} p_{i}+\ldots+\alpha_{j} p_{j}\right\}$. Let $\gamma_{\left\{p_{i} \cdots p_{j}\right\}}=\gamma p_{i} t_{i} \cdots p_{j} t_{j} \delta$ be such a circuit, where $\diamond t_{i}=\left\{p_{i}\right\}, \cdots, \diamond t_{j}=\left\{p_{j}\right\} \quad$ for the transitions $t_{i}, \cdots, t_{j}$ in $\gamma_{\left\{p_{i} \cdots p_{j}\right\}}$. Let $\sigma_{\left\{p_{i} \cdots p_{j}\right\}}=t_{i} \cdots t_{j}$ be the ordered sequence formed by the transitions in $\gamma_{\left\{p_{i} \ldots p_{j}\right\}}$. Clearly, $\sigma_{\left\{p_{i} \ldots p_{j}\right\}} \in \overline{L\left(B, M_{0}\right)}$, since the marking $M_{0}\left\{\alpha_{i} p_{i}+\ldots+\alpha_{j} p_{j}\right\}$ is enabling its firing. Since $\sigma_{\left\{p_{i} \ldots p_{i}\right\}}$ is a T-invariant (see Pag. 45, Lemma 3.9 in [4]) then, $\left(\sigma_{\left\{p_{i} \ldots p_{j}\right\}}\right)^{*} \in \overline{L\left(B, M_{0}\right)}$. By monotonicity, it is clear that both markings, $M_{0}\left\{\alpha_{i} p_{i}+\ldots+\alpha_{j} p_{j}\right\}+M_{0}^{\prime}\left\{\alpha_{k} p_{k}+\ldots+\alpha_{l} p_{l}\right\}$ and $2 M_{0}\left\{\alpha_{i} p_{i}+\ldots+\alpha_{j} p_{j}\right\}$, enables the firing of $\sigma_{\left\{p_{i} \ldots p_{i}\right\}}$. Consequently, there is an ambiguity about the marking reached by the firing of the transition sequence $\sigma_{\left\{p_{i} \ldots p_{i}\right\}}$. That is,

$$
\begin{aligned}
& \varphi\left(\mid M_{0}\left\{\alpha_{i} p_{i}+\ldots+\alpha_{j} p_{j}\right\}+\right. \\
& \left.\left.\quad+M_{0}^{\prime}\left\{\alpha_{k} p_{k}+\ldots+\alpha_{l} p_{l}\right\}\right)+B \vec{\sigma}_{\left\{p_{i} \ldots p_{j}\right\}}\right)= \\
& =\varphi\left(2 M_{0}\left\{\alpha_{i} p_{i}+\ldots+\alpha_{j} p_{j}\right\}\right)+\varphi\left(B \vec{\sigma}_{\left\{p_{i} \ldots p_{j}\right\}}\right)= \\
& =\varphi\left(2 M_{0}\left\{\alpha_{i} p_{i}+\ldots+\alpha_{j} p_{j}\right\}+B \vec{\sigma}_{\left\{p_{i} \ldots p_{j}\right\}}\right)
\end{aligned}
$$

Moreover, since $B \vec{\sigma}_{\left\{p_{i} \ldots p_{j}\right\}}=\overrightarrow{0}$, then, $\left(\sigma_{\left\{p_{i} \ldots p_{j}\right\}}\right)^{*}$ is always enabled. A contradiction to the $M D$ of the net.

(Sufficiency) Suppose that $\operatorname{ker} \varphi=\varnothing$. Then $\varphi$ is invertible. Thus, for any initial output of the system $y_{0}$ there exists a unique marking $M_{0}$ such that $M_{0}=\varphi^{-1} y_{0}$. Moreover, for every $y_{k}$ it is possible to compute $M_{k}=\varphi^{-1} y_{k}$ , as required.

Clearly, the Theorem 3 implies the Theorem 2. Thus, the following corollary holds.

Corollary 3. Let $\left(B, M_{0}, \varphi\right)$ be a non-safe OSS. Then, $\left(B, M_{0}, \varphi\right)$ is observable iff ker $\varphi=\varnothing$.

The following example illustrates the result.

Example 6. Consider the Figure 2. The Theorem 2 requires $E_{B}$ to be empty. Thus, subscripts have been added to $B_{1}, B_{2}$ and $C_{1}, C_{2}$. It is easy to note that $E_{B}$ is empty now. Accordingly, an observer is able to track any transitions sequence in the net. However, the extra token in $p_{1}$ is yet undiscoverable, since an observer is unable to decide in which of the places, either, $p_{1}, p_{3}$ nor $p_{7}$, the extra token remains. The Theorem 3 requires that $\varphi$ be of full range. Thus, subscripts have been added for sensor $A$, as well. It is easy to verify that now $\operatorname{ker} \varphi=\varnothing$. Notice that the Theorem 3 could be achieved with more efficient solutions, however, the optimal sensor placement is part of the future work.

If, for a particular problem the only interest is the Sequence-Detectability of a DES then, the Theorem 2 is the unique requirement, and accordingly, the Theorem 3 could be avoided.

\section{Conclusions}

This paper addressed the analysis of the sequence detection in DES modelled by PN's. The work is focused on well-formed OSS. The characterization of the property was firstly developed for safe OSS. Then, the safeness 
requirement was relaxed, and its implications in the sequence detection analyzed. The utility of the Sequence-Detectability in the testing of the observability of the OSS was analyzed. An example developed through the paper was used as an illustration of the main results. The future work includes the analysis of the relationship between the Myhill-Nerode theorem and the method for obtaining the SequenceDetectability table proposed in this paper.

\section{REFERENCES}

1. CAMPOS-RODRIGUEZ, R., M. ALCARAZ-MEJIA, A Matlab/Simulink Framework for the Design of Controllers and Observers for Discrete-Event Systems, Journal of Electronics and Electrical Engineering, vol. 99(3), 2010, pp. 63-68.

2. CAMPOS-RODRIGUEZ, R., M. ALCARAZ-MEJIA, J. MIRELESGARCIA, Supervisory Control of Discrete Event Systems Using Observers, Proc. of the IEEE Mediterranean Conference on Control and Automation, vol. 1, 2007, pp. 1-7.

3. CAMPOS-RODRIGUEZ, R., A. RAMIREZ-TREVINO, E. LOPEZMELLADO, Observability Analysis of Free-Choice Petri Net Models, Proceedings of IEEE/SMC Conference on Systems of Systems Engineering vol. 6, 2006, pp. 77-82.

4. DESEL, J., J. ESPARZA, Free Choice Petri Nets, Cambridge Univ. Press, 1995.

5. GIUA, A., C. SEATZU, Observability of Place / Transition Nets, IEEE Transactions on Automatic Control, vol. 47(9), 2002, pp. 1424-1437.

6. GIUA, A., C. SEATZU, F. BASILE, Observer-based State-feedback Control of Timed Petri Nets with Deadlock Recovery, IEEE Transaction on Automatic Control, vol. 49(1), 2004, pp. 17-29.

7. HERNANDEZ-MARTINEZ, E. G., E. S. PUGA-VELAZQUEZ, S. A. FOYOVALDES, J. A. MEDA-CAMPANA, Modeling Framework for Automated Manufacturing Systems Based on Petri Nets and ISA Standards, Studies in
Informatics and Control, vol. 22 (2), 2013, pp. 163-174.

8. HOPCROFT, J. E., R. MOTWANI, J. D. ULLMAN, Introduction to Automata Theory, Languages, and Computation, Addison-Wesley, 2001.

9. KAMMOUN, M. A., N. REZG, Z. ACHOUR, S. REZIG, State Space Search for Safe Time Petri Nets Based on Binary Decision Diagrams Tools: Application to Air Traffic Flow Management Problem, Studies in Informatics and Control, vol. 25(1), 2016, pp. 39-50.

10. KUMAR, R., M. A. SHAYMAN, Formulae Relating Controllability Observability and Co-observability, Automatica, 1998, pp. 211-215.

11. MINCA, E., A New Approach for the Control Optimization of an Assembly/Disassembly Mechatronics Line Served by an Autonomous Robotic System, Studies in Informatics and Control, vol. 23 (1), 2014, pp. 13-22.

12. MOODY, J. O., P. J. ANTSAKLIS, Petri Net Supervisors for DES with Uncontrollable and Unobservable Transitions, IEEE Transitions on Automatic Control, vol. 45(3), 2000, pp. 462-476.

13. MURATA, T., Petri Nets: Properties, Analysis and Applications, Proceedings of the IEEE, vol. 77(4), 1989, pp. 541-580.

14. OZVEREN, C. M., A. S. WILLSKY, Observability of Discrete Event Dynamic Systems, IEEE Transactions on Automatic Control, vol. 35(7), 1990, pp. 797-806.

15. OZVEREN, C. M., A. S. WILLSKY, Tracking and Restrictability in Discreteevent Dynamic Systems, SIAM Journal of Control and Optimisation, vol. 30(6), 1992, pp. 1423-1446.

16. RIVERA-RANGEL, I., A. RAMÍREZTREVIÑO, L. AGUIRRE-SALAS, J. RUIZ-LEON, Geometrical Characterization of Observability in Interpreted Petri Nets, Kybernetika, vol. 41(5), 2005, pp. 553-574.

17. SHU, S., F. LIN, H. YING, Detectability of Discrete Event Systems, IEEE 
Transactions on Automatic Control, vol. 52(12), 2007, pp. 2356-2359.

18. SHU, S., F. LIN, Detectability for Discrete Event Systems with Dynamic Event Observation, System Control Letters, vol. 59(1), 2010, pp. 9-17.

19. SHU, S., F. LIN, I-Detectability of Discrete-Event Systems, IEEE Transactions of Automatic Science and Engineering, vol. 10(1), 2013, pp. 187-196.
20. TAKAI, S., T. USHIO, S. KODAMA, Static-state Feedback Control of Discrete-event Systems under Partial Observation, IEEE Transaction on Automatic Control, vol. 40(11), 1995, pp. 1950-1954.

21. WONG, K. C., W. M. WONHAM, On the Computation of Observers in DiscreteEvent Systems, Discrete Event Dynamic Systems, vol. 14(1), 2004, pp. 55-107. 